DOI: 10.12731/2227-930X-2019-1-29-41

УДК 697.343

\title{
МАТЕМАТИЧЕСКАЯ МОДЕЛЬ УПРАВЛЕНИЯ ГИДРАВЛИЧЕСКИМИ РЕЖИМАМИ ТЕПЛОВОЙ СЕТИ
}

Липовка Ю.Л., Алексеев А.А.

Совершенствование методов управления гидравлическими режимами тепловых сети, в частности, управлением положения точки регулируемого давления (ТРД) является актуальной задачей, связанной с регулированием схемы подпитки тепловой сети, заключающейся в изменении положения ТРД путем дросселирования клапанами на байпасе сетевого (ииркуляционного) насоса, позволяюшие регулировать гидростатическое давление тепловой сети. В работе использована программа Solid works. Основным фактором, влияющим на положение линии гидростатического давления, является узел подпитки, состоящий из подпиточного насоса, регулирующих клапанов, датчика давления, расположенного в нейтральной точке.

Ключевые слова: тепловая сеть; пьезометрический график; гидростатические и гидродинамические режимь; нейтральная точка; точка регулируемого давления; характеристика сети, математическое моделирование.

\section{MATHEMATICAL MODEL OF MANAGEMENT OF HYDRAULIC MODES OF THE HEATING NETWORK}

\author{
Lipovka Y.L., Alekseev A.A.
}

Improving the management of the hydraulic modes of the heat network, in particular, controlling the position of the regulated pressure point (TRD) is an important task related to regulating the heating net- 
work make-up scheme, which consists in changing the position of the turbofan engines by throttling valves on the bypass of the network (circulation) pump, which allows hydrostatic pressure of the heat network. The work uses the program Solid works. The main factor affecting the position of the hydrostatic pressure line is a make-up unit consisting of a make-up pump, control valves, and a pressure sensor located at the neutral point.

Keywords: heat network; piezometric graph; hydrostatic and hydrodynamic regimes; neutral point; regulated pressure point; network characteristic, mathematical modeling.

\section{Введение}

В связи с тем, что независимая, закрытая схема подключения тепловой сети является основной схемой подключения жилых и нежилых зданий, то становится актуально использовать возможность регулирования гидростатического давления пьезометрического графика, без негативных последствий.

В настоящее время отсутствует методика плавного регулирование положения гидростатического режима тепловой сети. Привязка тепловой сети к изменению гидростатического режима, позволит принять новую, улучшенную модель подпиточного узла.

В конце 20 века Ионин А.А. предложил, что степень закрытия клапанов на байпасной линии подбирается, таким образом, чтобы в задвижке расположенной после циркуляционного насоса, полный напор равнялся $H_{\text {ст }}=H_{\text {нач }}-\Delta H_{\mathrm{A}}$ где $\Delta H_{\mathrm{A}}-$ потери давления на задвижке расположенной после ЦН, а в задвижке расположенной до

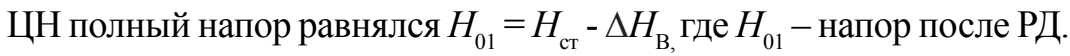

Разработка улучшенной модели подиточного узла позволит решить ряд вопросов связанные с нехваткой давления потребителя, избежать установки станций повышения давления, увеличением протяженности тепловой сети.

При увеличение гидростатического давление, мы получаем преимущества, по сравнение с простым увеличением частоты оборотов сетевого насоса. Система остается стабильно устойчивой, не 
изменяются потери давления по длине и местных сопротивлениях, не изменяется скорость в сечении.

Для управления гидравлическими режимами необходимо использование определенного метода гидравлического расчета. В статье [1] рассмотрены вопросы анализа потокораспределения в регулируемых трубопроводных системах. Предложено в систему уравнений метода «глобального градиента» ввести отметки рельефа местности и пьезометрические напоры, что позволяет в процессе итеративного приближения оценивать гидравлические режимы тепловых сетей с насосными подстанциями, регуляторами расхода и давления. Крайне важным в гидравлических расчетах, особенно кольцевых систем является плавность поведения математической модели, описывающей коэффициент гидравлического трения. Авторами [2] выполнен сравнительный анализ многих известных формул для определения коэффициента гидравлического трения в трубах с точки зрения точности и скорости расчета. Для обеспечения плавного перехода от ламинарного режима к переходному в критической зоне предложен алгоритм кубической интерполяции общего вида.

Простота использования моделирования локальной потребности в теплоте, изучение применимости одной и той же методики моделирования к тематическим исследованиям с различной плотностью населения предложено в [3]. В [4] представлена упрощенная модель распределения тепла от производственных предприятий до потребителей. Суть заключается в уменьшение или сокращение магистральных тепловых сетей. Авторами [5] разрабатывается методология поиска наилучшей стратегии расширения сети централизованного теплоснабжения при наличии ряда ограничений. Используя смешанный целочисленный подход к линейному программированию, разработанная модель оптимизирует будущую работу энергетического центра путем выбора наилучшего сочетания технологий для достижения определенной цели. Предложено, что управление тепловых сетей в статье [6] заключается в повышение эффективности подстанции централизованного теплоснабжения благодаря новой стратегии управления. 
Работа [7] основана на имитационном анализе температур подачи воды во вторичной сети, которые получены в результате моделирования эжектора по сравнению с температурами подачи воды, которое использует предприятие с теплообменниками. Кроме того, результаты, полученные при моделировании CFD, показали значительный потенциал экономии тепловой энергии.

\section{Материалы и методы исследования}

Рассмотрен процесс влияния дросселирующих клапанов на положение ТРД и предложен графический метод определения ТРД. Данный метод позволит сравнить результаты, полученные путем гидравлического расчета, с результатами, представленными на графической зависимости, также метод позволит оценить влияния регулирующих устройств на положение пьезометрического графика.

Нейтральная точка - точка в которой давление поддерживается постоянным. Нейтральная точка, располагается на перемычке статичного трубопровода сетевого насоса, как показано на рисунке 1.

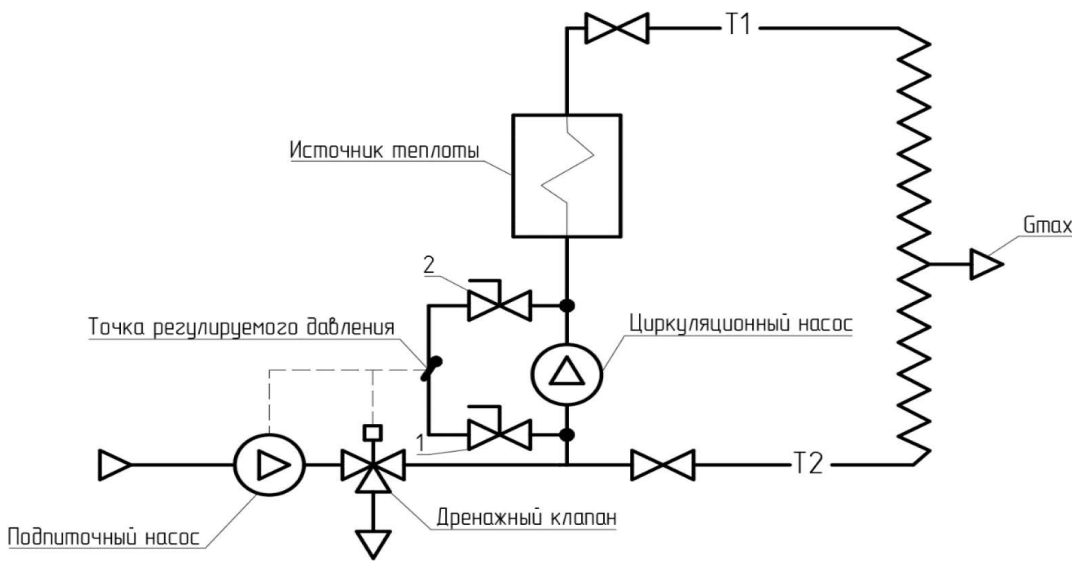

Рис. 1. Принципиальная схема системы теплоснабжения

Fig. 1. Schematic diagram of the heat network

Принцип работы регулирующих устройств при гидродинамическом режиме: при увеличении утечки теплоносителя из сети, 
давление в нейтральной точке начинается снижаться, клапан регулятор давления приоткрывается, увеличивается подпитка тепловой сети и давление восстанавливается. При прекращении утечки, давление в нейтральной точке начинает повышаться и клапан регулятора давления прикрывается. Если при закрытом клапане давление будет продолжать расти, то в работу включится дренажный клапан, поддерживающий постоянное давление в нейтральной точке, и произойдет сброс избытка воды в емкость.

Статическое давление регулируется с помощью подпиточного насоса.

Используя регулирующую арматуру на байпасе, можно регулировать гидростатическое давление тепловой сети. Использования регулирования гидростатического режима тепловой сети, заключается в экономии затрат связанных на подключение дополнительных потребителей.

Процесс регулирования гидростатического давления заключается в изменении положения пьезометрического графика, с помощью положения нейтральной точки. При закрытии регулирующего вентиля расположено до сетевого насоса, давление в нейтральной точке будет равно давлению, развиваемому после циркуляционного насоса, следовательно, сработает датчик давления расположенный на нейтральной точке, датчик отправит сигнал об увеличении давления на дренажный клапан и клапан откроется дросселируя избыток давления, тем самым гидростатическое давление сместится по оси ординат до запрограммированного давления на нейтральные точки. При закрытии регулирующего вентиля расположенного после циркуляционного насоса, давление в нейтральной точке будет равняться давлению на всасе сетевого насоса, датчик давления расположенный на нейтральной точке отправит сигнал о понижении давления на регулятор давления и подпиточный насос, тем самым насос начнет заполнять систему до заданного значения, следовательно, гидростатическое давления начнет смещаться вверх параллельно оси ординат.

Принцип работы нейтральной точки описан на рисунке 3,4. 


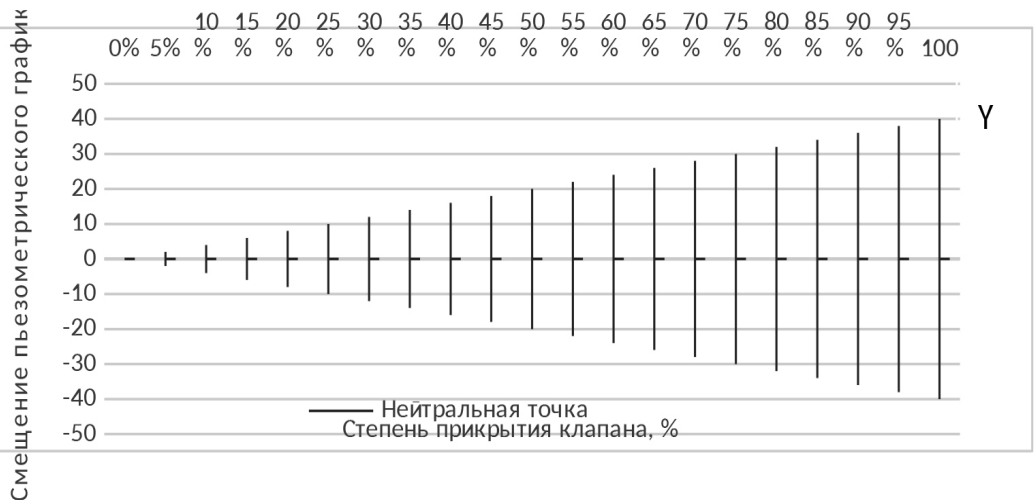

Рис. 2. Графическая зависимость нейтральной точки от регулирующих вентилей

Fig. 2. Graphic dependence of the neutral point on the control valves

Графическая зависимость позволяет определить точку плавного регулирования относительно нейтрального (нейтральной точки) положения пьезометрического напора. На ординате гидродинамического режима показан процесс возможного отклонения гидростатического режима тепловой сети относительно степени прикрытия регулирующей арматуры.

По оси ординат наблюдается положение нейтральной точки.

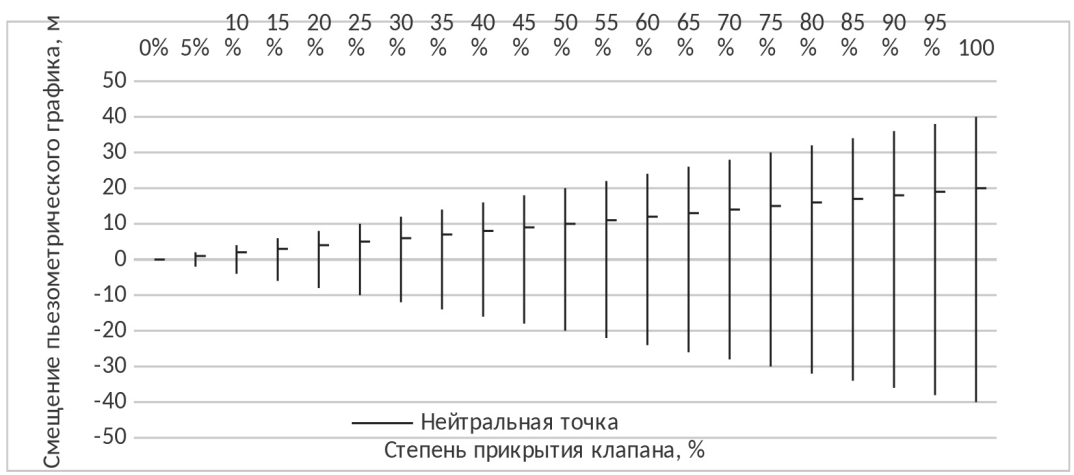

Рис. 3. Смещение нейтральной точки при закрытии регулирующего вентиля №2 на 50\%

Fig. 3. Neutral point shifts when closing control valve No. 2 is $50 \%$ closed 
На рисунке 3 показан процесс смещения гидростатического напора относительно степени прикрытия клапана, возможное максимальное (расчетное) отклонение 40 м.вод.ст по оси ординат от нулевого положения.

Использование уточненной методики гидравлического расчета [1], позволит принять расчетные параметры на байпасе.

\section{Результаты исследования}

Влияние клапанов на пьезометрический график подпиточного узла. Подпиточный насос развивает напор $H_{\text {ст. }}$ Напор на байпасной

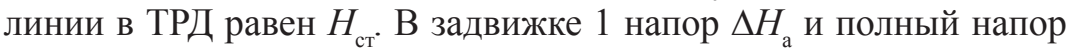
после нее рассчитывается, как $H_{01}=H_{\text {нач }}-\Delta H_{\text {РК2 }}$. В задвижке 2 напор $H_{02}=H_{\text {рд1 }}-\Delta H_{\text {РК1 }}$. Регулятор давления поддерживает постоянное давление в точке ТРД между регуляторами давления 1 и 2 , при этом в точке после РД2 будет поддерживаться напор $\mathrm{H}_{01}$.

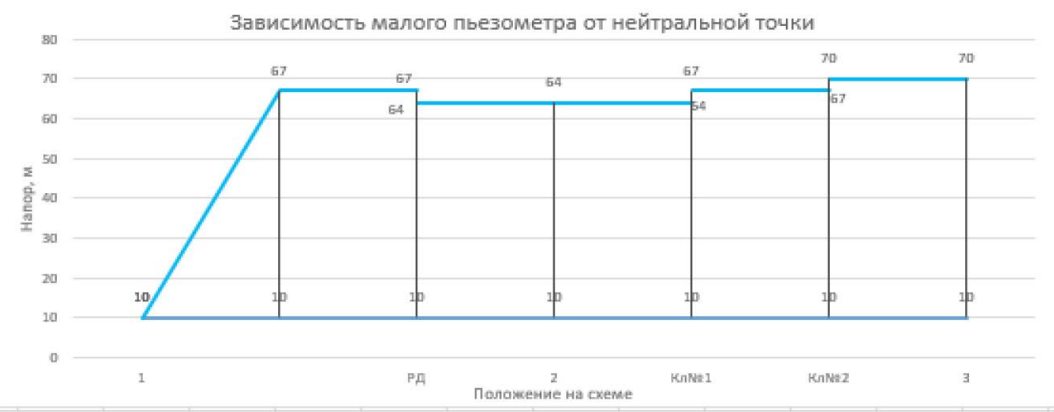

Pис. 4. Пьезометрический график узла подпитки в нейтральном положении Fig. 4. Piezometric graph of the feed station in neutral position

Вычислительная динамика жидкости, CFD - Computational Fluid Dynamics, является разделом механики жидкости, определяющаяся как набор методологий, которая позволяет компьютеру предоставить нам численное моделирование потоков жидкости. Анализ CFD может использоваться для распределения скорости, давления и температуры через байпас. Расчетная модель позволяет сравнить полученные результаты с графической зависимостью 
нейтральной точки от РД рис. 2. Инструменты компьютерного моделирования, такие как CFD, предоставляют гораздо более глубокие возможности для моделирования потока жидкости, а также используются при моделировании различных ситуаций.

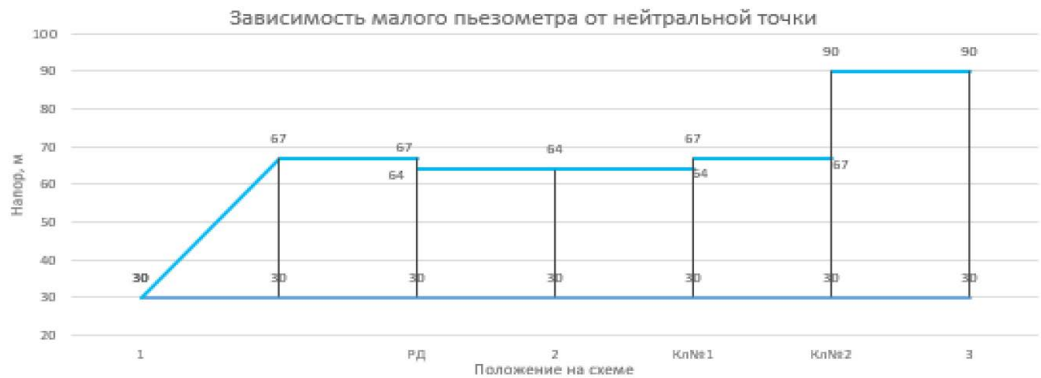

Рис. 5. Пьезометрический график узла подпитки при закрытии клапана №2 на $50 \%$ Fig. 5. Piezometric graph of the make-up assembly when valve No. 2 is closed by $50 \%$

Основой для расчета одномерного потока в напорных трубопроводах является система дифференциальных уравнений, состоящая из импульса, непрерывности и уравнения теплового потока. Уравнения потока могут быть записаны в следующем виде с использованием переменных давления р и расхода L (1),(2).

$$
\begin{gathered}
\frac{\partial L}{\partial t}+\frac{L}{A} \cdot \frac{\partial L}{\partial x}-\frac{L}{A} \cdot \frac{\partial A}{\partial p} \cdot \frac{\partial p}{\partial t}+\frac{L^{2}}{A^{2}}+g \cdot A \cdot \frac{\partial p}{\partial x}+g \cdot A \cdot S_{F}-a_{x} \cdot A=0 \\
\frac{1}{p \cdot a^{2}} \cdot\left(A \cdot \frac{\partial p}{\partial t}+L \cdot \frac{\partial p}{\partial x}\right)+\frac{\partial L}{\partial x}-\frac{L}{A} \cdot \frac{\partial A}{\partial x}=0
\end{gathered}
$$

где $g=$ ускорение свободного падения;

$z=$ координата;

$\rho=$ плотность;

$x=$ расчетная координатная ось;

$t=$ время;

$a=$ ускорение жидкости

$a_{x}=$ дополнительное ускорение в направлении х.

Использование уравнений, представленных выше, не может дать точных результатов. Поэтому в данной работе методы CFD 
использовались для моделирования потока жидкости в определенной среде. Анализ CFD проводился со следующими допусками: поток несжимаемый и однородный, отсутствие теплопередачи, коэффициент шероховатости равен 0 (не учитывался), анализ проводился в устойчивом состоянии.

\section{Обсуждение}

Моделировались следующие условия жидкости: температура воды в первичной сети $\mathrm{T} 1=150{ }^{\circ} \mathrm{C}$, статическое давление воды в первичной сети $\mathrm{P} 1=4$ бар, расход воды $\mathrm{L}=215 \mathrm{~m} 3 /$, диаметр магистрального трубопровода $\mathrm{d}=300 \mathrm{Mм}$, диаметр байпаса $\mathrm{d}_{\sigma}=80 \mathrm{Mм}$, плотность воды $\rho=1000$ кг $/ \mathrm{M}^{3}$, вязкость воды $\mu=1.003 \mathrm{e}-3$ Па·с

Модель CFD показана на рис. 6.

На рисунке 6 точки, обозначенные Т1...-Т11 - расчетная точка принятая в программе solid works.

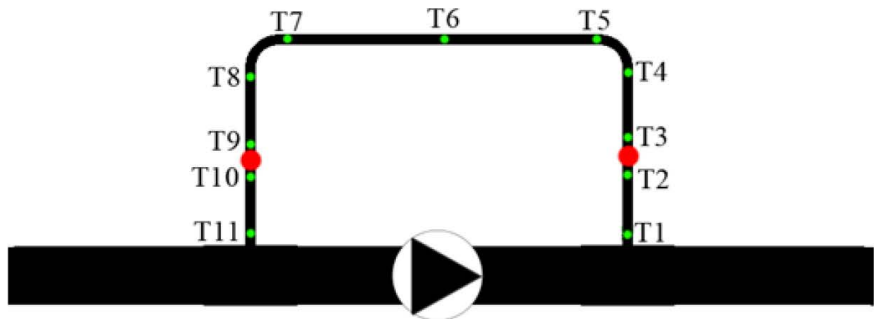

Рис. 6. Точечные параметры

Fig. 6. Point options

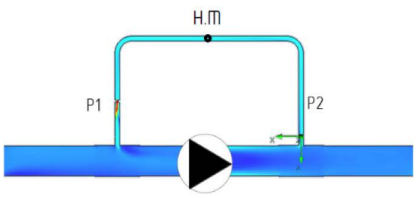

$\mathrm{H} 1$

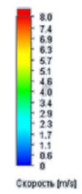

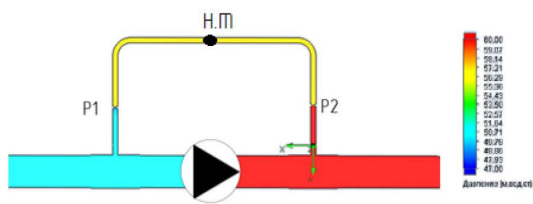

$\mathrm{H1}$

Рис. 7. Скорость потока при прикрытии клапана №1 и №2 на $30 \%$

Fig. 7. Flow rate when covering valve No. 1 and No. 2 on $30 \%$

На рисунке 7 прикрыт регулирующий вентиль $\mathrm{P} 1$ и Р2, давление в нейтральной точке (ТРД) определяется по формуле 3. 


$$
\text { Рн.T }=\frac{P 2+P 1}{2}
$$

Гидростатическое давления, будет плавно возрастать, до расчетного значения. Присутствует возможность плавного регулирования гидростатического давления тепловой сети.
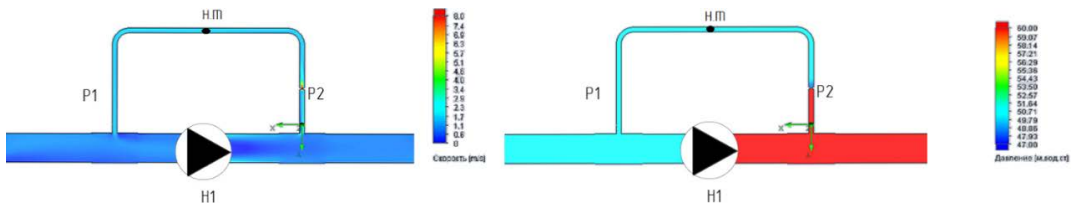

Рис. 8. Скорость потока при прикрытии клапана №2

Fig. 8. Flow rate when covering valve No. 2

На рисунке 8 прикрыт регулирующий клапан, расположенный после нагнетателя. давление в нейтральной точке (ТРД) определяется по формуле 4.

$$
\text { PH.T }=\mathrm{P}_{2}-\frac{P 1}{100}
$$

Гидростатическое давления при прикрытие регулирующего клапана, распложённого после нагнетателя, будет плавно возрастать, до расчетного значения, недостаток данного регулирования, отсутствие плавного регулирования.

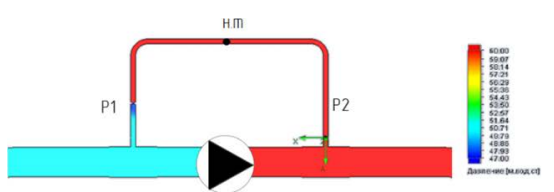

H1

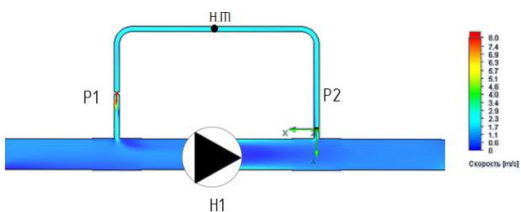

H1

Pис. 9. Скорость потока при прикрытии клапана №1

Fig. 9. Flow velocity when covering valve No. 1

На рисунке 9 прикрыт регулирующий клапан, расположенный до нагнетателя, давление в нейтральной точке (ТРД) определяется по формуле 5.

$$
\text { PH.T }=\mathrm{P}_{1}-\frac{P 2}{100} .
$$

Гидростатическое давления при прикрытие регулирующего клапана, останется низменным, данный метод регулирования не 
позволяет нам добиться нужных результатов. Также был произведен расчет при прикрытии клапанов на $56 \%, 80 \%, 100 \%$. Полученные данные представлены на рисунке 11, 12.

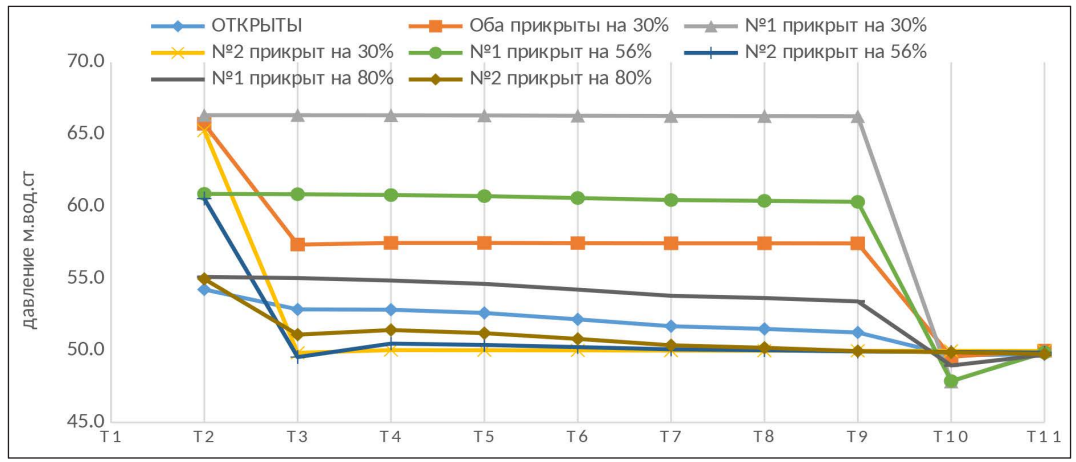

Рис. 11. Точечные параметры давления

Fig. 11. Point options preassure

На рисунке 11 давление в каждой точке, зависит от потерь давления на клапане. При совместной работе РД возможно более плавное регулирование гидростатического режима.

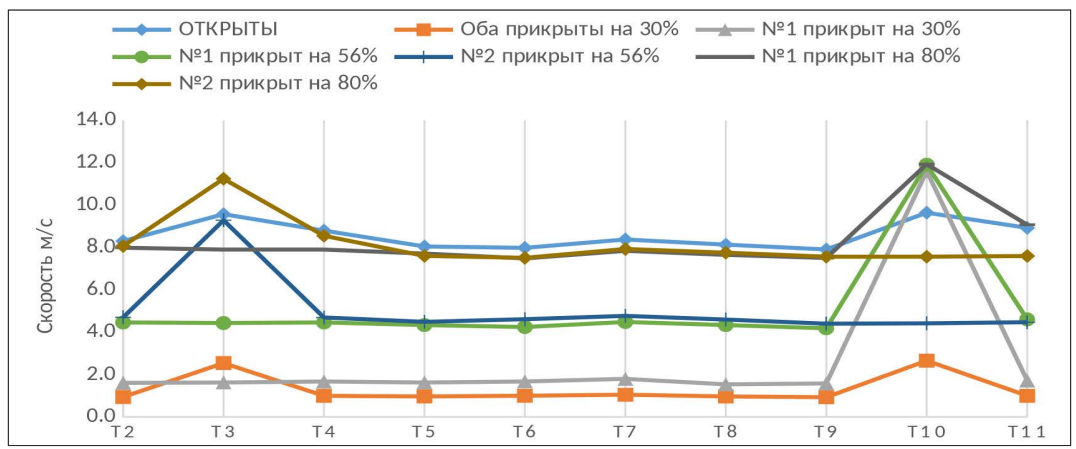

Pис. 12. Точечные параметры скорости

Fig. 12. Point options speed

На рисунке 12 указана скорость в каждой расчетной точке согласно рисунка 9, во всех случаях график имеет очень высокие скачки в точке Т10, исключением является совместная работа обоих клапанов. 


\section{Заключение}

Расчеты, полученные в программе solid works показываю, что совместная работа РД является самым оптимальным решением в регулирование гидростатического режима.

На рисунке 3 показан процесс изменения положения гидростостатического режима. Изменяя положение пьезометрического графика, мы не влияем на другие параметры теплой сети, а это позволит снизить потребляемую нагрузку на циркуляционный насос в случае увеличение протяженности трассы. Почему увеличение числа оборотов насоса не приведет к должному результату увеличения напора системы теплоснабжения, потому что расход и напор имеет зависимость $\mathrm{f}(\mathrm{L} ; \mathrm{P})$ при увеличении напора насоса, увеличивается и расход, исходя из формулы дарси, потери давления имеют прямую квадратичную зависимость от скорости.

Установка подпиточных станций на трассе теплоснабжения имеет колоссальные затраты на их монтаж и обслуживание. Основной недостаток метода управления ТРД является то, что он актуален только для закрытой системы теплоснабжения.

Поэтому изучения ТРД в закрытой системе теплоснабжения, позволит сэкономить огромные денежные средства за счёт управления потокораспределением, именно на этом малом узле, влияющем кардинально на работу всей системы.

Авторы заявляют об отсутствии конфликта интересов.

Исследование не имело спонсорской поддержки.

\section{Список литературы / References}

1. Lipovka A.J., Lipovka Y.L. Application of «Gradient» Algorithm to Modeling Thermal Pipeline Networks with Pumping Stations // Journal of Siberian Federal University. 1 Engineering \& Technologies (2013 6) 28-35.

2. Lipovka A.J., Lipovka Y.L. Determining Hydraulic Friction Factor for Pipeline Systems // Journal of Siberian Federal University. 1 Engineering \& Technologies (2014 1), 62-82.

3. Brocklebank I., Beck S.B.M., Styring P. A. simple approach to modeling rural and urban district heating // Frontiers in Energy Research. №6. Issue OCT. 15 October 2018. № 103. 
4. Larsen H.V., Pálsson H., Bøhm B., Ravn H.F. Aggregated dynamic simulation model of district heating networks // Energy Conversion and Management, 43 2002. N.8. C. 995-1019.

5. Delangle A., Lambert R.S.C., Shah N., Acha S., Markides C.N. Modelling and optimising the marginal expansion of an existing district heatingnetwor// EnergyVolume N140.2017. C. 209-223.

6. Gustafsson J., Delsing J., van Deventer J. Improved district heating substation efficiency with a new control strategy //Applied Energy Volume N87. Issue 6. June 2010. C. 1996-2004.

7. Berisha X., Meha D. Modelling of direct connection of heat consumers to district heating with ejectors // International Journal on Advanced Science, Engineering and Information Technology. №8. Issue 1. 2018.C. 219-226.

\section{ДАННЫЕ ОБ АВТОРАХ}

Липовка Юрий Львович, доктор технических наук

Сибирский федеральный университет, инженерно-строительный институт

пр. Свободный, 79, г. Красноярск, 660041, Российская Федеращия

\section{Алексеев Алексей Андреевич, аспирант}

Сибирский федеральный университет, инженерно-строительный институт

пр. Свободный, 79, г. Красноярск, 660041, Российская Федеращия Alexeev.alexey.ru@mail.ru

\section{DATA ABOUT THE AUTHORS}

Lipovka Yuri Lvovich, Doctor of Technical Sciences

Siberian Federal University, Institute of Civil Engineering 79, Svobodny pr., Krasnoyarsk, 660041Ю Russian Federation

Alekseev Alexey Andreevich, graduate student

Siberian Federal University, Institute of Civil Engineering 79, Svobodny pr., Krasnoyarsk, 660041Ю Russian Federation Alexeev.alexey.ru@mail.ru

ORCID: 0000-0001-5078-7364 